\title{
Estimation of the Gini coefficient for the lognormal distribution of income using the Lorenz curve
}

\author{
Kwasi A. Darkwah*, Ezekiel N. N. Nortey and Anani Lotsi
}

*Correspondence:
kwasidarkwah88@yahoo.com
Department of Statistics,
School of Physical
and Mathematical Sciences,
College of Basic and Applied
Sciences, University of Ghana,
Accra, Ghana

Accra, Ghana

\begin{abstract}
The main objective of the study is to compare the Newton-Cotes methods such as the Trapezium rule, Simpson 1/3 rule and Simpson 3/8 rule to estimate the area under the Lorenz curve and Gini coefficient of income using polynomial function with degree 5. Comparing the Gini coefficients of income computed from the Polynomial function with degree 5 for the Trapezium, Simpson 1/3 and Simpson 3/8 methods using the relative errors showed that the trapezium rule, Simpson's $1 / 3$ rule and Simpson's 3/8 rule show negative biases with the Simpson $1 / 3$ rule yielding the lowest absolute relative true error of $4.230711 \%$.
\end{abstract}

Keywords: Lorenz curve, Gini coefficient, Newton-Cotes methods, Lognormal distribution, Income distribution

\section{Background}

The concept of inequality is broad as compared to poverty. This is because the definition of inequality is based on the whole population and not only a section of the population below a definite poverty line. Several measures of inequality must satisfy six basic criteria to become an accepted measure of inequality. They are; statistical testability, mean independence, Pigou-Dalton transfer sensitivity, population size independence, symmetry and decomposability (Glewwe 1986).

The simple inequality measure of a population separates those who are poor from those who are rich and shows the percentage of income ascribed to every quintile or decile of the population. The quintile which shows those who are poor are mostly $6-10 \%$ of all income and the highest quintile fall within 35-50\%. Gini (1912) and Bassmann et al. (1990) have all devised various ways of measuring income inequality. The importance of measuring income on national basis is to ensure an efficient management of the financial and economic policies that will ensure price stability and economic growth.

According to Edozien (1991), poor health, illiteracy, unemployment and poverty are some characteristics of people who receive low income while literacy, stable jobs, sufficient health care are the characteristics of high income earners. 
The size of income inequality is mostly measured and interpreted by the Lorenz curve. The Lorenz curve is a graph that accounts for the cumulative percentage of household income to the cumulative percentage of income received when the households are put in ascending order of their income.

Gini coefficient is one of the most efficient and commonly used measurement of income inequality in the world. It is computed as twice the region between a Lorenz curve and the egalitarian line. Gini coefficient as a measure of inequality is widely used in various contexts such as energy, credit availability, income, health care and wealth (Berndt et al. 2003).

This indeed confirms Morgan (1962) statement that the Gini index is the best single measure of inequality. The Gini coefficient is analyzed based on discrete and continuous distributions (Yitzhaki and Schechtman 2005). Golden (2008) demonstrated how estimates of Gini coefficient can be computed using numerical integration method.

Fellman (2012) estimated the Gini coefficient of income by employing numerical integration methods such as the Trapezium rule and Simpson's rule. His research revealed that the Trapezium rule yield positive biases for the Lorenz curve while the Gini coefficient yielded negative biases.

Darkwah et al. (2016a) recently used numerical integration methods such as Boole and Weddle methods to compare the Gini coefficient of Ghanaian income from the 2013 Ghana Living Standard Survey (GLSS6) which were estimated using Rasche et al. (1980) function and polynomial function. Their results revealed that the estimated area under the Lorenz curve and Gini coefficients using Rasche et al. (1980) function and polynomial function according to the Boole and Weddle method of integration resulted in positive and negative biases respectively but the Weddle's method was better as compared to Boole method of numerical integration in estimating the Gini coefficient of income with the Boole method producing the highest absolute relative error.

The main objective of the study is to compare the Newton-Cotes methods such as the Trapezium rule, Simpson 1/3 rule and Simpson 3/8 rule to estimate the area under the Lorenz curve and Gini coefficient of income using polynomial function with degree 5.

\section{Methods}

All data used in this study was based on secondary data from the Ghana Living Standards Survey (GLSS6). The data had a total income of GhC 244,759,213.2 from a total of 58,788 individuals.

Let the vector $z=\left(z_{1}, z_{2}, z_{3}, \ldots, z_{n}\right)$ denote the distribution of individual income in a population with $i=1,2,3, \ldots, n$ and $n$ is the total number of individuals. The income distribution has mean and density function as $\mu(z)$ and $f(z)$ respectively. The Lorenz curve and Gini index of income for the lognormal distribution is given as $\Phi\left(\Phi^{-1}(z)-\sigma\right)$ and $2 \Phi(\sigma / \sqrt{2})-1$ respectively where $\sigma$ is the standard deviation and $\Phi(z)$ is the standard normal distribution (Cowell 1995).

Suppose $\pi(z)$ shows the cumulative proportion of the individuals that receive income up to $\mathrm{z}$ and $\eta(z)$ shows the cumulative proportion of the total income that is received by individuals in the same population. The Lorenz curve shows the relationship that exists between $\pi$ and $\eta$. The Lorenz curve is established by letting $z$ be the income of every individual and $f(z)$ be the probability density function of $z$. Hence 


$$
\pi(z)=\int_{0}^{z} f(y) d y
$$

and

$$
\eta(z)=\frac{1}{\mu} \int_{0}^{z} y f(y) d y
$$

The mean of the distribution of income is;

$$
\mu=\int_{0}^{\infty} y f(y) d y
$$

Ryu and Slottje (1996) declared that a function is fitted to the Lorenz curve in other to estimate the area under the Lorenz curve. A polynomial function proposed by Becker and Weispfenning (1993) is chosen from the several functions suggested for estimating the area under the Lorenz curve. Becker and Weispfenning (1993) suggested a polynomial which is a mathematical expression involving a sum of powers in one or more variables multiplied by coefficients to estimate the area under the Lorenz curve. A univariate polynomial with constant coefficients is given by;

$$
\eta(\pi)=a_{m} \pi^{m}+a_{m-1} \pi^{m-1}+\cdots+a_{1} \pi+a_{0}
$$

When $a_{m} \neq 0$ and $m \geq 2$, the polynomial function is a continuous non-linear function.

The area under the Lorenz curve was estimated using a polynomial function with degree 5 .

The estimate of the Lorenz curve is given by

$$
L(\pi)=\int_{0}^{1} \eta(\pi) d \pi
$$

The Gini coefficient which measures the inequality among the distribution of a nation's residential income is given by;

$$
\text { Gini }=1-2 \int_{0}^{1} \eta(\pi) d \pi
$$

The random variable $Z$ is modelled using the lognormal distribution with probability density and cumulative distribution function of $f_{\left(\mu, \sigma^{2}\right)}(z)=\frac{1}{z \sqrt{2 \pi \sigma^{2}}} e^{-\frac{(\operatorname{In} z-\mu)^{2}}{2 \sigma^{2}}}, z>0, \sigma>0, \mu>0$ and $F(z)=\phi\left(\frac{\operatorname{In} z-\mu}{\sigma}\right)$ respectively, where $\operatorname{In} z$ is normally distributed. The mean of $Z$ is $E(z)=e^{\mu+\sigma^{2} / 2}$ and the variance of $Z$ is $V(z)=\left(e^{\sigma^{2}}-1\right) e^{2 \mu+\sigma^{2}}$. 
The estimated parameters of the Lognormal distribution function from the Ghana household data on income would be integrated using the Newton-Cotes method such as the Trapezium rule, Simpson's 1/3 rule and Simpson 3/8 rule to compute the Gini coefficient. The Newton-Cotes method involves $n$ points in the interval $[a, b]$ with $n-1$ order polynomial which passes through points $z_{i}$ and are equally spaced. Approximating the area under the curve $y=f(z)$ from $z=a$ to $z=b$, using the Trapezium rule, Simpson 1/3 rule and Simpson 3/8 rule with $z_{0}=a, z_{n}=b$ and $h=\frac{(b-a)}{n}$ is (Sauer 2012; Darkwah et al. 2016b; Mettle et al. 2016).

For Trapezoidal rule, we have;

$$
A=\frac{h}{2}\left[f\left(z_{0}\right)+f\left(z_{1}\right)\right]
$$

For Simpson 1/3 rule, we have;

$$
A=\frac{h}{3}\left[f\left(z_{0}\right)+4 f\left(z_{1}\right)+f\left(z_{2}\right)\right]
$$

For Simpson 3/8 rule, we have;

$$
A=\frac{3 h}{8}\left[f\left(z_{0}\right)+3 f\left(z_{1}\right)+3 f\left(z_{2}\right)+f\left(z_{3}\right)\right]
$$

The relative errors are used to compared the Trapezium rule, Simpson 1/3 rule and Simpson 3/8 rule employed to estimate the Gini coefficients of the various regions, rural and urban areas, male and female heads. The relative error is computed as;

$$
\varepsilon_{a}=\frac{\text { Absolute Errors }}{\text { Exact }}
$$

Where Absolute Error $=$ Exact - Approximate and the number of significant digits at least correct is given as;

$$
m=2-\log \left(\frac{\left|\varepsilon_{a}\right|}{0.02}\right)
$$

The bias is calculated as;

$$
\text { Bias }=\text { Approximate }- \text { Exact }
$$

The Romberg numerical integration was used to calculate the "exact" numerical integration method. A general expression for Romberg integration can be written as (Sauer 2012; Darkwah et al. 2016b);

$$
I_{2 n}^{(k)}=\frac{4^{k} I_{2 n}^{(k-1)}-I_{n}^{(k-1)}}{4^{k-1}-1}, \quad k \geq 2
$$

The index $\mathrm{k}$ represents the order of extrapolation and $I_{n}^{(1)}$ represents the values obtained from the regular trapezoidal rule with $\mathrm{n}$ intervals. Using $k=2$ which represents values obtained using the true estimate as $o\left(h^{2}\right)$. Hence

$$
I_{\text {true }} \cong I_{\text {true }, \text { est }}=I_{2 n}+\frac{I_{2 n}-I_{n}}{3}
$$


The main objective of this study is to compare the Gini coefficients of income computed using the Trapezium rule, Simpson 1/3 rule and Simpson 3/8 rule.

\section{Results}

To apply the methodology proposed by this study, data on gross income was taken from the sixth round of the Ghana Living Standard Survey (GLSS 6) conducted by the Ghana Statistical Service (GSS). The data comprises of a nationwide sample of 58,788 family size with a total income of GHC $244,759,213.2$. The mean and standard deviation of the Ghanaian income are $14,788.18$ and $46,257.611$ respectively. With a maximum income of $2,184,471$ and a minimum income of 2 , a substantial difference exists between the maximum and minimum income values which is an indication of a very high disparity in the income levels of Ghanaians. Also, skewness of 16.709 and kurtosis of 479.105 show that the data deviates widely from the normal distribution. The Kolmogorov-Smirnov (KS) test was used to test whether the distribution of the income is lognormal. The null hypothesis of the data following a lognormal distribution, produced a $\mathrm{p}$ value of 0.42 greater than 0.05 which is consistent with the lognormal.

Consequently, the total income $(z)$ is modeled using the lognormal distribution with parameters $\mu$ and $\sigma^{2}$. The density function of the income which follows the lognormal distribution is given by

$$
f_{\left(\mu, \sigma^{2}\right)}(z)=\frac{1}{z \sqrt{2 \pi \sigma^{2}}} e^{-\frac{(I n z-\mu)^{2}}{2 \sigma^{2}}}, \quad z>0, \quad \sigma>0, \quad \mu>0
$$

The maximum likelihood estimators of the parameters $\mu$ and $\sigma^{2}$ of the lognormal distribution based on the income distribution are given as $\hat{\mu}=\frac{\sum_{i} I n z_{i}}{n}=8.5311$ and $\hat{\sigma}^{2}=\frac{\sum_{i}\left(I n z_{i}-\hat{\mu}\right)^{2}}{n}=1.45812$.

The cumulative distribution function of the income is also given by;

$$
F(z)=\phi\left(\frac{\operatorname{In} z-8.5311}{1.45812}\right)
$$

The Kolmogorov-Smirnov test which is used to test whether the distribution of income for the various regions, rural and urban areas and family heads is lognormal are shown in Table 1. Also the estimated Gini coefficient of the lognormal distribution can also be found in Table 1 .

From Table 1, all the p values of the Kolmogorov-Smirnov tests of the various regions, rural and urban areas, male and female family heads are greater than 0.05 which is consistent with the lognormal. Also from Table 1, the estimates of the Gini index for lognormal distribution was found to be between 0.63174 and 0.68234 with respect to the regions where Greater Accra and Upper East region recorded the lowest and highest income inequality measured using the Gini index for the lognormal distribution.

Given $\pi(z)$ to be the proportion of the units that receive income up to $z$ and $\eta(z)$ to represent the proportion of total income received by the same units whose income are less than or equal to $z$, then using the Polynomial function with degree 5 to compute the estimates of the area under the Lorenz curve for nonlinear income distribution, the 
Table 1 Kolmogorov-Smirnov test, estimates of the mean, standard deviation and Gini index for lognormal distribution of the Ghanaian income

\begin{tabular}{|c|c|c|c|c|c|}
\hline \multirow[t]{2}{*}{ Region } & \multicolumn{2}{|c|}{ Kolmogorov-Smirnov test } & \multirow{2}{*}{$\begin{array}{l}\text { Estimated } \\
\text { mean }\end{array}$} & \multirow{2}{*}{$\begin{array}{l}\text { Estimated standard } \\
\text { deviation }\end{array}$} & \multirow[t]{2}{*}{ Gini index } \\
\hline & Statistic & p value & & & \\
\hline Greater Accra & 0.0313 & 0.18486 & 8.7613 & 1.2724 & 0.63174 \\
\hline Eastern & 0.0241 & 0.2353 & 8.6814 & 1.3380 & 0.6559 \\
\hline Ashanti & 0.021 & 0.3548 & 8.7915 & 1.3570 & 0.66272 \\
\hline Volta & 0.0291 & 0.1964 & 8.5908 & 1.3640 & 0.6652 \\
\hline Western & 0.0257 & 0.2471 & 9.0076 & 1.3462 & 0.65886 \\
\hline Brong Ahafo & 0.0244 & 0.2733 & 8.5370 & 1.3702 & 0.6674 \\
\hline Central & 0.0219 & 0.4305 & 8.1159 & 1.2877 & 0.63746 \\
\hline Northern & 0.0217 & 0.4014 & 8.3369 & 1.3222 & 0.65018 \\
\hline Upper West & 0.0342 & 0.06939 & 7.8074 & 1.3215 & 0.64992 \\
\hline Upper East & 0.031 & 0.1381 & 8.1671 & 1.4132 & 0.68234 \\
\hline Rural & 0.0276 & 0.2226 & 8.2519 & 1.2533 & 0.6245 \\
\hline Urban & 0.0305 & 0.1102 & 7.2546 & 1.3411 & 0.65702 \\
\hline Male head & 0.0271 & 0.2251 & 8.2114 & 1.3252 & 0.65128 \\
\hline Female head & 0.0269 & 0.2462 & 8.2322 & 1.4021 & 0.67852 \\
\hline All & 0.0221 & 0.4201 & 8.5311 & 1.45812 & 0.69748 \\
\hline
\end{tabular}

estimate of the area under the Lorenz curve using the Trapezium rule, Simpson 1/3 rule and Simpson 3/8 rule are shown in Table 2.

The estimate of the Gini coefficient of income using the estimate of the area under the Lorenz curve from the Trapezium rule, Simpson 1/3 rule and Simpson 3/8 rule is shown in Table 3 below.

The computation of the Biases and Relative errors using the Trapezium, Simpson 1/3 and Simpson 3/8 numerical integration method is shown in Table 4.

Table 2 Estimates of the area under the Lorenz curve using Trapezium, Simpson 1/3 and Simpson 3/8 numerical integration methods

\begin{tabular}{lllll}
\hline Region & Trapezium rule & Simpson's 1/3 rule & Simpson's 3/8 rule & Exact integration \\
\hline Greater Accra & 0.2764 & 0.2684 & 0.2704 & 0.2623 \\
Eastern & 0.2675 & 0.2593 & 0.2626 & 0.2523 \\
Ashanti & 0.2655 & 0.2599 & 0.2613 & 0.2517 \\
Volta & 0.2690 & 0.2622 & 0.2656 & 0.2562 \\
Western & 0.2428 & 0.2426 & 0.2427 & 0.2370 \\
Brong Ahafo & 0.2632 & 0.2593 & 0.2611 & 0.2165 \\
Central & 0.2633 & 0.2603 & 0.2612 & 0.2553 \\
Northern & 0.2379 & 0.2319 & 0.2328 & 0.2304 \\
Upper East & 0.2296 & 0.2196 & 0.2235 & 0.2095 \\
Upper West & 0.3126 & 0.2246 & 0.2278 & 0.2143 \\
Rural & 0.2566 & 0.2530 & 0.2533 & 0.2513 \\
Urban & 0.2754 & 0.24465 & 0.2475 & 0.2258 \\
Male head & 0.2702 & 0.2613 & 0.2619 & 0.26 \\
Female head & 0.2618 & 0.23525 & 0.2389 & 0.2171 \\
All & 0.2660 & 0.2483 & 0.2504 & 0.23855 \\
\hline
\end{tabular}


Table 3 Estimates of Gini coefficient using the Trapezium, Simpson 1/3 and Simpson 3/8 numerical integration methods

\begin{tabular}{lllll}
\hline Region/family head & Trapezium rule & Simpson's 1/3 rule & Simpson's 3/8 rule & Exact integration \\
\hline Greater Accra & 0.4472 & 0.4632 & 0.4592 & 0.4754 \\
Eastern & 0.4650 & 0.4814 & 0.4748 & 0.4954 \\
Ashanti & 0.4690 & 0.4802 & 0.4774 & 0.4966 \\
Volta & 0.4620 & 0.4756 & 0.4688 & 0.4876 \\
Western & 0.5144 & 0.5148 & 0.5146 & 0.526 \\
Brong Ahafo & 0.4736 & 0.4814 & 0.4778 & 0.567 \\
Central & 0.4734 & 0.4794 & 0.4776 & 0.4894 \\
Northern & 0.5242 & 0.5362 & 0.5344 & 0.5392 \\
Upper East & 0.5408 & 0.5608 & 0.5530 & 0.581 \\
Upper West & 0.3748 & 0.5508 & 0.5444 & 0.5714 \\
Rural & 0.4868 & 0.4940 & 0.4934 & 0.4974 \\
Urban & 0.4492 & 0.5107 & 0.5050 & 0.5484 \\
Male head & 0.4596 & 0.4774 & 0.4762 & 0.48 \\
Female head & 0.4764 & 0.5295 & 0.5222 & 0.5658 \\
All & 0.4680 & 0.5034 & 0.4992 & 0.5229 \\
\hline
\end{tabular}

Table 4 Biases generated from the Gini coefficient using Trapezium, Simpson 1/3 and Simpson 3/8 numerical integration methods as compared with the exact estimation

\begin{tabular}{llll}
\hline Region/family head & Trapezium rule & Simpson's 1/3 rule & Simpson's 3/8 rule \\
\hline Greater Accra & -0.0282 & -0.0122 & -0.0162 \\
Eastern & -0.0304 & -0.014 & -0.0206 \\
Ashanti & -0.0276 & -0.0164 & -0.0192 \\
Volta & -0.0256 & -0.012 & -0.0188 \\
Western & -0.0116 & -0.0112 & -0.0114 \\
Brong Ahafo & -0.0934 & -0.0856 & -0.0892 \\
Central & -0.016 & -0.01 & -0.0118 \\
Northern & -0.015 & -0.003 & -0.0048 \\
Upper East & -0.0402 & -0.0202 & -0.028 \\
Upper West & -0.1966 & -0.0206 & -0.027 \\
Rural & -0.0106 & -0.0034 & -0.004 \\
Urban & -0.0992 & -0.0377 & -0.0434 \\
Male head & -0.0204 & -0.0026 & -0.0038 \\
Female head & -0.0894 & -0.0363 & -0.0436 \\
All & -0.0549 & -0.0195 & -0.0237 \\
\hline
\end{tabular}

From Table 5 below, the highest relative error of $34.40672 \%$ was computed using the Trapezium numerical integration method for the Upper West Region while the lowest relative error of 0.55638 \% was computed from the Simpson 1/3 numerical integration method for the Northern Region. Also, the highest relative error for the family heads was $15.80064 \%$ for female family head computed from the Trapezium rule while the lowest relative error for the family heads was $0.541667 \%$ for male family head computed from the Simpson 1/3 rule. The Urban area had the highest relative error of $18.08899 \%$ from the Trapezium rule while the Simpson 1/3 rule was used to compute the lowest relative error of $0.683554 \%$ for rural area. 
Table 5 Relative Error generated from the Gini coefficient using Trapezium, Simpson 1/3 and Simpson 3/8 numerical integration methods as compared with the exact estimation

\begin{tabular}{lllllll}
\hline Region/family head & $\begin{array}{l}\text { Trapezium } \\
\text { Relative error (\%) }\end{array}$ & $\begin{array}{l}\text { Rule } \\
\mathbf{m}\end{array}$ & $\begin{array}{l}\text { Simpson's 1/3 } \\
\text { Relative error (\%) }\end{array}$ & $\begin{array}{l}\text { Rule } \\
\mathbf{m}\end{array}$ & $\begin{array}{l}\text { Simpson's 3/8 } \\
\text { Relative error (\%) }\end{array}$ & $\begin{array}{l}\text { Rule } \\
\mathbf{m}\end{array}$ \\
\hline Greater Accra & 5.931847 & 2 & 2.566260 & 2 & 3.407657 & 2 \\
Eastern & 6.136455 & 1 & 2.825999 & 2 & 4.158256 & 2 \\
Ashanti & 5.557793 & 2 & 3.302457 & 2 & 3.866291 & 2 \\
Volta & 5.250205 & 2 & 2.461034 & 2 & 3.855619 & 2 \\
Western & 2.205323 & 2 & 2.129278 & 2 & 2.167300 & 2 \\
Brong Ahafo & 16.47266 & 1 & 15.09700 & 1 & 15.73192 & 1 \\
Central & 3.269309 & 2 & 2.043318 & 2 & 2.411116 & 2 \\
Northern & 2.781899 & 2 & 0.556380 & 2 & 0.890208 & 2 \\
Upper East & 6.919105 & 1 & 3.476764 & 2 & 4.819277 & 2 \\
Upper West & 34.40672 & 1 & 3.605180 & 2 & 4.725236 & 2 \\
Rural & 2.131082 & 2 & 0.683554 & 2 & 0.804182 & 2 \\
Urban & 18.08899 & 1 & 6.874544 & 1 & 7.913931 & 1 \\
Male head & 4.25000 & 2 & 0.541667 & 2 & 0.791667 & 7.705903 \\
Female head & 15.80064 & 1 & 6.415695 & 1 & 2 & 4.532415 \\
All & 10.49914 & 1 & 3.729203 & & 2 \\
\hline
\end{tabular}

\section{Conclusion}

The main aim of the study was to compare the estimates of the Gini coefficient of income computed using the Trapezium rule, Simpson 1/3 rule and Simpson 3/8 rule. The results showed that positive biases were generated for the area under the Lorenz curve for all the Newton-Cotes methods such as the Trapezium rule, Simpson 1/3 rule and Simpson $3 / 8$ rule used while from Table 4, the Gini coefficient generated negative biases. This shows that there is no uniform optimization for the Newton-Cotes methods used. From Table 5, the Trapezium method generated the highest relative error of $34.6583 \%$ for the Upper West Region while the lowest relative error of 0.81391 \% was computed from the Simpson 1/3 method for the Northern Region. Also, using the entire income data, the Trapezium rule yielded the highest relative error of 10.97626 \% while the Simpson $1 / 3$ rule yielded the lowest relative error of $4.230711 \%$. This shows that the Simpson's 1/3 is the best method compared to Trapezium rule and Simpson 3/8 rule in estimating the Gini coefficient of income. This confirms Darkwah et al. (2016a) and Fellman (2012) findings that the area under the Lorenz curve and Gini coefficient yields negative and positive biases. Also, Trapezium rule, Simpson 1/3 rule and Simpson 3/8 rule applied to the Polynomial of order 5 gave lesser estimates of the Gini coefficient of income when compared to the estimates of the Gini index for the lognormal distribution in Table 1. Future research could use other Newton-Cotes methods such as Boole and Weddle applied to other Lorenz curve functions such as Gupta (1984) and Ortega et al. (1991) to estimate the Gini coefficient and compare them to the lognormal distribution estimates of the Gini coefficient. It is also recommended to investors, policy makers, financial analysts and stakeholders in the world that when choosing Newton-Cotes methods to compute the inequality of income of their countries, they should make use of the Simpson 1/3 numerical integration method instead of the Trapezium rule and Simpson 3/8 rule. 


\section{Authors' contributions}

KAD conceptualized the methodology of the study and also took part in the analysis. ENNN worked on the literature review and also took part in the data analysis. CAL wrote some part of the discussion of the manuscript. All authors agree to be accountable for all aspects of the work. All authors read and approved the final manuscript.

\section{Acknowledgements}

Special appreciation is extended to the Carnegie Corporation of New York for funding this research under the New Generation of Academics in Africa through the Office of Research, Innovation and Development, University of Ghana.

\section{Competing interests}

The authors declare that they have no competing interests.

Received: 27 January 2016 Accepted: 19 July 2016

Published online: 28 July 2016

\section{References}

Bassmann R, Hayes K, Johnson J, Slottje D (1990) A general functional form for approximating the Lorenz curve. J Econom 43:77-90

Becker T, Weispfenning V (1993) A computational approach to commutative algebra. Graduate Text in Mathematics 141 , Springer, New York

Berndt DJ, Fisher JW, Rajendrababu RV, Studnicki J (2003) Measuring health care inequities using the Gini index. System sciences. In: Proceedings of the 36th annual Hawaii international conference on IEEE

Cowell FA (1995) Measuring inequality, 2nd edn. Harvester Wheatsheaf, Hemel Hempstead

Darkwah KA, Nortey ENN, Mettle FO, Baidoo I (2016a) A study of the estimation of the Gini coefficient of income using Lorenz curve. Br J Math Comput Sci 15(4). ISSN:2231-0851

Darkwah KA, Nortey ENN, Lotsi CA (2016b) A proposed numerical integration method using polynomial interpolation. Br J Math Comput Sci 16(2): 1-11. ISSN:2231-0851

Edozien EC (1991) Inequality and poverty issue in Concept and Thorny. The Nigeria Economic Society, Nigeria

Fellman J (2012) Estimation of Gini coefficients using Lorenz curves. J Stat Econom Methods 1(2):31-38

Gini C (1912) Measurement of inequality of incomes. Econ J 31:124-126

Glewwe P (1986) The distribution of Income in Sri Lanka in 1969-70 and 1980-81: a decomposition analysis. J Dev Econ $24: 255-274$

Golden J (2008) A simple geometric approach to approximating the Gini coefficient. J Econ Educ 39(1):68-77

Gupta MR (1984) Functional form for estimating the Lorenz curve. Econometrica 52:1313-1314

Mettle FO, Quaye EN, Aseidu L, Darkwah KA (2016) A proposed method for numerical integration. Br J Math Comput Sci $17(1): 1-15$. ISSN:2231-0851

Morgan J (1962) The anatomy of income distribution. Rev Econ Stat 44:270-283

Ortega P, Martin A, Fernandez A, Ladoux M, Garcia A (1991) A new functional form for estimating Lorenz curves. Rev Income Wealth 37:447-452

Rasche RH, Gaffney J, Koo AY, Obst N (1980) Functional forms for estimating Lorenz curves. Econometrica 48:1061-1062

Ryu HK, Slottje DJ (1996) Two flexible functional form approaches for approximating the Lorenz curve. J Econom $72: 251-274$

Sauer T (2012) Numerical analysis, 2nd edn. George Mason University. Pearson Education Inc, Upper Saddle River. ISBN-10:0321783670

Yitzhaki S, Schechtman E (2005) The properties of the extended Gini measures of variability and inequality. Metron Int J Stat LXIII(3):401-433

\section{Submit your manuscript to a SpringerOpen ${ }^{\oplus}$ journal and benefit from:}

Convenient online submission

\section{- Rigorous peer review}

- Immediate publication on acceptance

- Open access: articles freely available online

- High visibility within the field

- Retaining the copyright to your article

Submit your next manuscript at springeropen.com 